\title{
Peer support for mental illness in India: an underutilised resource
}

\author{
S. Pathare*, J. Kalha and S. Krishnamoorthy \\ Centre for Mental Health Law and Policy, Indian Law Society, Pune, India
}

First published online 5 April 2018

Key words: Community mental health, health service research, other psychosocial techniques/treatments, social support.

\section{Introduction}

Peer support involves the provision of support and services to persons with mental illness by individuals who have a current or past experience of mental health problems (Davidson et al. 2006). Peer support is based on the idea that a person who has experience of a mental health problem is better placed to empathise and understand the difficulties and barriers to recovery of another. Mutual sharing and peer modelled learning may help promote self-efficacy and recovery (Salzer \& Shear, 2002). There are many types of peer support ranging from informal peer support groups, which are loosely structured at one end to a more professionalised form where peer support workers are employed by mental health services and work alongside other mental health professionals (Lloyd-Evans et al. 2014).

While peer support workers are increasingly employed in mental health services in high-income countries such as Australia (Franke et al. 2010), New Zealand (Scott, 2011), UK (Repper \& Carter, 2011), USA (Lloyd-Evans et al. 2014) and Europe (Castelein et al. 2008), they are nearly absent in low- and middle-income countries such as India. In this article, we will outline the mental health system in India, recent mental health reforms in India and a description of introducing peer support workers as part of a quality improvement project in mental health services in Gujarat, India.

\section{Mental health burden and treatment gap in India}

India's population as of 2011 was 1.2 billion people, with a total of 35 States/Union Territories, 642 districts, 5924 sub-districts, 7935 towns and 640867 villages. In 2005, the National Commission on Macroeconomic and Health estimated that 1-2\% (10-20 million, in 2005) of

\footnotetext{
* Address for correspondence: S. Pathare, Centre for Mental Health Law and Policy, Indian Law Society, Pune, India.

(Email: spathare@cmhlp.org)
}

the Indian population had severe mental disorders, and an estimated 5\% (50 million, in 2005) had mild-to-moderate mental disorders irrespective of severity, yielding an overall estimated prevalence rate of $6.5 \%$ (National Commission on Macroeconomics \& Health, 2005). A more recent national epidemiological survey (Gururaj et al. 2016) estimated overall weighted current prevalence of $10.6 \%$ and lifetime prevalence of $13.7 \%$ for any mental morbidity (including alcohol and substance abuse). This epidemiological survey also estimated a treatment gap of $73.6 \%$ for severe mental illness and of $85.0 \%$ for common mental disorders. There is a huge shortage of trained mental health resources according to a reply by the Ministry of Health \& Family Welfare in Parliament in December 2015, there are 3800 psychiatrists, 898 clinical psychologists, 850 psychiatric social workers and 1500 psychiatric nurses nationwide (Salve, 2016).

\section{Innovations in mental health service delivery}

Acknowledging this huge disparity between need and (non-) availability of trained mental health human resources, task sharing and task shifting to non-mental health professionals and non-health professionals such as lay workers, care givers and 'affected individuals' (peer workers) have been proposed as the only viable immediate solutions to address the huge treatment gap (Kakuma et al. 2011). The District Mental Health Programme (DMHP) of the Government of India relies upon non-specialist health professionals such as primary health care doctors to deliver mental health services but has been criticised for underperforming and failing to meet mental health needs particularly in rural areas (Goel, 2011). Large randomised controlled trials from India have also demonstrated the efficacy of lay health workers for depression (Patel et al. 2010; 2017) and alcohol use (Nadkarni et al. 2017), but these have not yet been implemented at scale across the health system. Other task-sharing initiatives in 
India include using community-based volunteers to provide mental health and social care (ShieldsZeeman et al. 2017), which is being implemented at scale in one district (two million population) and undergoing evaluation in a stepped-wedge cluster randomised trial (personal communication). Although India has a vibrant innovation culture around task shifting/task sharing, it is worth noting that there have been no attempts until recently (see below) to use peer support to improve access to mental health services.

\section{Mental health policy and law reform}

Since 2010, India has embarked on mental health policy reform, leading to the development of India's first National Mental Health Policy in 2014 (New Pathways, New Hope: National Mental Health Policy of India, 2014) and the Mental Health Care Act in 2017 (Government of India, 2017).

The National Mental Health Policy is based on values and principles of equity, justice, integrated and evidence-based care, quality, participatory and holistic approach to mental health. Policy goals include reducing distress, disability, exclusion, morbidity and premature mortality associated with mental health problems across life span of a person; enhance understanding of mental health in the country; provide universal access to mental health care; increase access to mental health services for vulnerable groups; reduce risk and incidence of suicide and attempted suicide; ensure respect for rights and protection from harm of persons with mental health problems; and reduce stigma associated with mental health problems and enhance availability and distribution of skilled human resources for mental health (New Pathways, New Hope: National Mental Health Policy of India, 2014).

The policy acknowledges the shortage of trained mental health human resources and recognises the need for 'appropriately trained lay and communitybased counsellors', but peer support or peer support workers do not find mention in the national policy. The policy emphasises the need to 'foster recovery' and highlights the need for community-based rehabilitation programmes and centres and supporting families in promoting recovery. India's mental health policy thus conceptualises recovery as clinical recovery getting rid of symptoms, improved social functioning and being 'normal' while internationally there is increasing focus on personal recovery, which is seen as a unique personal journey including the development of new meaning and purpose for one's life despite mental illness (Slade et al. 2008). Peer support is an essential part of personal recovery focused on mental health services (Scottish Recovery Network, 2005; Slade, 2013) but not necessarily of services focused only on clinical recovery. This may explain the absence of peer support services in India's mental health policy.

The Mental Health Care Act (Government of India, 2017) passed by the Parliament in 2017 is a landmark legislation for India in many respects. Although India does not have a legislated right to healthcare, the Act now mandates a right to access mental health care. Thus, uniquely amongst all countries, India has a legislated right to mental health care but not a right to health care: the significance of this has to be understood in mental health sector's battle for parity with physical health care across the world. The Act outlines a minimum package of services that must be provided by the Government to fulfil this right. However, this minimum package does not mention peer support or peer support services and only includes professional mental health services using a medical paradigm. The Act also mandates parity for mental health care with physical health care in both public and private health insurance.

The Act is also the first healthcare legislation in India to introduce advance directives for mental health care. Any person, whether they have a mental illness or not, can write an advance directive stating the treatment they wish to have, the treatment they do not wish to have and also appoint a nominated representative to make decisions on their behalf if necessary. The advance directive provision has been criticised mainly by psychiatrists as not being evidence based (Sarin et al. 2012), challenges in implementation in India (Thara \& Rameshkumar, 2012) and fears that it will drive a wedge between service users and their families (Kala, 2013). There are recent studies from India showing that service users, including those with psychotic symptoms, are able to complete advance directives with little assistance and appreciated the opportunity to exercise control over treatment of their mental illness in a future episode of decisional incapacity (Kumar et al. 2013; Shields et al. 2013; Pathare et al. 2015). Peer support workers employed by health services and appropriately trained can be a potential resource to assist service users in understanding and completing advance directives, thus addressing a potential barrier that health professionals may not have time in busy clinical practice to do so (Shields et al. 2014).

\section{Peer support volunteers as part of the QualityRights project in Gujarat}

In 2014, Grand Challenges Canada funded a transition to scale implementation project - QualityRights 
Gujarat (Mental Health Innovation Network: QualityRights Gujarat, 2015). The project, based on the WHO's QualityRights programme (Funk \& Drew, 2017), was implemented at nine public mental health facilities in the western State of Gujarat (population approximately 60 million), India. Gujarat has four public mental hospitals and ten mental health units in public general hospitals, serving roughly 280 000 persons annually in 2014. As part of the project, peer support volunteers (PSVs) were introduced in six public mental health facilities for the first time in mental health services in India. PSVs in this instance are individuals with lived experiences of mental illness who assist other service users with similar experiences in enabling their personal recovery by helping them in preparing recovery plans. They also organise weekly peer support groups (Maitri groups) at their respective mental health facilities. PSVs are trained in recovery-oriented care and basic communication skills. PSVs are paid a small honorarium to cover their travel and expenses (approximately US $\$ 50$ per month) and have flexible working hours. Initially, PSVs were supported by project funds. One of the key achievements towards PSV sustainability was the provision of financial resources by the State Mental Health Authority, Government of Gujarat since June 2016. There is a current funding provision for 35 PSVs with a plan to increase their numbers over time. In meetings with project staff, PSVs have reported increased empowerment and self-confidence. A formal qualitative evaluation of peer support by PSVs has been carried out and the data are being analysed and will be published shortly.

The PSVs in their role approached other service users who were regular at the facility, listened to and empathised with their current experience, while also sharing their personal narratives with them. The use of recovery plan as a tool assisted in structuring a conversation with service users at the facility and provided the communication link between service users and health care professionals. One of the PSVs at an international conference in India reported that 'knowledge about recovery has not only developed confidence within me, but also the confidence to help other service users', and that 'this project has given me a sense of hope and has strengthened my belief that I can do better'. Another PSV reflected that 'one thing was clear that medication only helped in partial recovery. But selfhelp and self - help groups are also very important for a patient to live a normal life'. The role provided a platform to the PSVs to move towards their personal goals, such as enter professional employment space which was previously denied to them.

The mere presence of PSVs in public mental health facilities made the system believe that service users have agency. The implementation of PSV model created a space for conversation around the validity and purpose of their role within a tertiary care facility. One of the key spaces for the same was through peer support group meetings, which were initiated, planned and organised by PSVs themselves.

\section{Challenges to peer support in India}

There are numerous challenges to implementing peer support in routine mental health services in India. As mentioned earlier in this paper, the National Mental Health Policy is not oriented to personal recovery and hence does not provide policy support to introducing peer support workers in mental health services. The absence of policy support makes it difficult to convince policy makers to allocate funds to establishing peer support services.

We are unable to identify any efficacy or effectiveness studies on peer support in India, and this lack of local evidence base is a significant barrier to convincing policy makers. Qualitative findings from QualityRights Gujarat as described above will hopefully encourage other local governments in India to invest in peer support services.

India is a hierarchal society and mental health services, like other health services in India, follow a medical model with the power imbalances in the doctor-patient relationship (Fochsen et al. 2006). The hierarchal nature of the doctor-patient relationship means that professional knowledge and opinion of the doctors is given primacy, while clients and their opinions are seen as subordinate (Shields et al. 2013). This has implications for introducing peer support in mental health services - for example, whether psychiatrists and other mental health professionals will support peer support in clinical services if peer support workers are perceived as exerting independent influence on their service users remains an unanswered question. We also do not know whether service users in India and their care givers will perceive peer support as 'healthcare' and utilise such services.

To bring a paradigm shift in the power imbalance mentioned above, peer support needs systemic implementation at institutional level as well as in community settings. The lack of infrastructure further implies lack of validated spaces for service users to advocate for their rights. The dominance of a medical model and under-resourced setting of mental health care impose the need on peer support to replicate the elements of a medical model rather than validate the unique contribution of peer support in personal recovery. The lacuna in conceptual clarity on the role of peer support vis-a-vis other mental health care professionals 
and the absence of a structure for professional growth for peer supporters add further barriers to integrate a recovery-oriented approach. We need an active service user movement that can catalyse the growth of peer support services to implement peer support within clinical and community-based services.

Extended families in India play an important role in providing practical, material and emotional support to their family members with mental illness. This leads to a narrative that is not of the service users themselves but curated by the caregiver or health care provider. This reduces the perceived need by individuals for support from peers and simultaneously a discouragement by families to seek support from outside the family. A final challenge is the absence of structured training programmes and locally contextualised training manuals to train persons with lived experience in peer support work.

\section{Conclusions}

Although recent policy reform in India falls short in directly supporting the development of peer support services, the implementation of new law and experience from initial attempts to incorporate peer support in mental health services in Gujarat represents a potential opportunity. Policy acceptance of task-shifting/tasksharing paradigm also provides another opportunity to emphasise the role that peer support can play in improving access to mental health support and services and thus reduce treatment gap. Finally, there is a need for well-designed quantitative and qualitative studies from India to create an evidence base for peer support.

\section{Acknowledgement}

We would like to thank the entire team of QualityRights Gujarat, Department of Health and Family Welfare, Government of Gujarat, Peer Support Volunteers, heads of mental health facilities, health care professionals, and caregivers.

\section{References}

Castelein S, Bruggeman R, van Busschbach JT, van der Gaag M, Stant AD, Knegtering H, Wiersma D (2008). The effectiveness of peer support groups in psychosis: a randomized controlled trial. Acta Psychiatrica Scandinavica 118, 64-72.

Davidson L, Chinman M, Sells D, Rowe M (2006). Peer support among adults with serious mental illness: a report from the field. Schizophrenia Bulletin 32, 443-450. https://doi. org/10.1093/schbul/sbj043.

Fochsen G, Deshpande K, Thorson A (2006). Power imbalance and consumerism in the doctor-patient relationship: health care providers' experiences of patient encounters in a rural district in India. Qualitative Health Research 16, 1236-1251. https://doi.org/10.1177/ 1049732306293776.

Franke CCD, Paton BC, Gassner LAJ (2010). Implementing mental health peer support: a South Australian experience. Australian Journal of Primary Health 16, 179-186.

Funk M, Drew N (2017). WHO qualityrights: transforming mental health services. The Lancet Psychiatry 4, 826-827. https://doi.org/10.1016/S2215-0366(17)30271-7.

Goel DS (2011). Why mental health services in low- and middle-income countries are under-resourced, underperforming: an Indian perspective. National Medical Journal of India 24, 94-97.

Government of India (2017). Mental Health Care Act, 2017. Act 10 of 2017. http://lawmin.nic.in/ld/P-ACT/2017/ A2017-10.pdf.

Gururaj G, Varghese M, Benegal V, Rao GN, Pathak K, Singh LK, Mehta RY, Ram D, Shibukumar TM, Kokane A, Lenin Singh RK, Chavan BS, Sharma P,

Ramasubramanian C, Dalal PK, Saha PK, Deuri SP, Giri AK, Kavishvar AB, Sinha VK, Thavody J, Chatterji R, Akoijam BS, Das S, Kashyap A, Ragavan VS, Singh SK, Misra R and NMHS collaborators group (2016). National Mental Health Survey of India, 2015-16: Mental Health Systems. National Institute of Mental Health and Neuro Sciences, Bengaluru.

Kakuma R, Minas H, van Ginneken N, Dal Poz MR, Desiraju K, Morris JE, Saxena S, Scheffler RM (2011). Human resources for mental health care: current situation and strategies for action. The Lancet 378, 1654-1663. https:// doi.org/10.1016/S0140-6736(11)61093-3.

Kala A (2013). Time to face new realities; mental health care bill-2013. Indian Journal of Psychiatry 55, 216. https://doi.org/ 10.4103/0019-5545.117129.

Kumar TR, John S, Gopal S, Mohan G, Joseph J, Rangaswamy T (2013). Psychiatric advance statements: an Indian experience. International Journal of Social Psychiatry 59, 531-534. https://doi.org/10.1177/0020764012443756.

Lloyd-Evans B, Mayo-Wilson E, Harrison B, Istead H, Brown E, Pilling S, Johnson S, Kendall T (2014). A systematic review and meta-analysis of randomised controlled trials of peer support for people with severe mental illness. BMC Psychiatry 14, 39. https://doi.org/10. 1186/1471-244X-14-39.

Mental Health Innovation Network: QualityRights Gujarat (2015). Accessed on 3 February 2018 http://www. mhinnovation.net/innovations/qualityrights-gujarat.

Nadkarni A, Weobong B, Weiss HA, McCambridge J, Bhat B, Katti B, Murthy P, King M, McDaid D, Park AL, Wilson GT, Kirkwood B, Fairburn CG, Velleman R, Patel V (2017). Counselling for alcohol problems (CAP), a lay counsellor-delivered brief psychological treatment for harmful drinking in men, in primary care in India: a randomised controlled trial. The Lancet 389, 186-195. https:// doi.org/10.1016/S0140-6736(16)31590-2.

National Commission on Macroeconomics and Health (2005). Report of the National Commission on Macroeconomics and Health. Ministry of Health and Family Welfare, New Delhi, India. 
New Pathways, New Hope: National Mental Health Policy of India 2014. New Delhi India. Accessed on 3 February 2018 https://www.nhp.gov.in/sites/default/files/pdf/national\% 20mental\%20health\%20policy\%20of\%20india\%202014.pdf.

Patel V, Weiss HA, Chowdhary N, Naik S, Pednekar S, Chatterjee S, De Silva MJ, Bhat B, Araya R, King M, Simon G, Verdeli H, Kirkwood BR (2010). Effectiveness of an intervention led by lay health counsellors for depressive and anxiety disorders in primary care in Goa, India (MANAS): a cluster randomised controlled trial. The Lancet 376, 2086-2095. https://doi.org/10.1016/S0140-6736(10) 61508-5.

Patel V, Weobong B, Weiss HA, Anand A, Bhat B, Katti B, Dimidjian S, Araya R, Hollon SD, King M, Vijayakumar L, Park AL, McDaid D, Wilson T, Velleman R, Kirkwood BR, Fairburn CG (2017). The healthy activity program (HAP), a lay counsellor-delivered brief psychological treatment for severe depression, in primary care in India: a randomised controlled trial. The Lancet 389, 176-185. https://doi.org/10. 1016/S0140-6736(16)31589-6.

Pathare S, Shields L, Nardodkar R, Narasimhan L, Bunders J (2015). What do service users want? A content analysis of what users may write in psychiatric advance directives in India. Asian Journal of Psychiatry 14, 52-56. https://doi.org/10. 1016/j.ajp.2014.10.006

Repper J, Carter T (2011). A review of the literature on peer support in mental health services. Journal of Mental Health 20, 392-411. https://doi.org/10.3109/09638237.2011. 583947.

Salve P (2016). Nearly 60 Million Indians Suffer From Mental Disorders. IndiaSpend-Journal. Accessed on 3 February 2018 http://www.indiaspend.com/cover-story/nearly-60million-indians-suffer-from-mental-disorders-68507.

Salzer MS, Shear SL (2002). Identifying consumer-provider benefits in evaluations of consumer-delivered services. Psychiatric Rehabilitation Journal 25, 281-288.
Sarin A, Murthy P, Chatterjee S (2012). Psychiatric advance directives: potential challenges in India. Indian Journal of Medical Ethics 9, 104-107. https://doi.org/10.20529/IJME. 2012.031.

Scott A (2011). Authenticity work: mutuality and boundaries in peer support. Society and Mental Health 1, 173-184. https://doi.org/10.1177/2156869311431101.

Scottish Recovery Network (2005). The role and potential development of peer support services. Accessed on 3 February 2018 https://www.scottishrecovery.net/ wp-content/uploads/2005/12/Peer-support-briefing.pdf.

Shields LS, Pathare S, van Zelst SD, Dijkkamp S, Narasimhan L, Bunders JG (2013). Unpacking the psychiatric advance directive in low-resource settings: an exploratory qualitative study in Tamil Nadu, India. International Journal of Mental Health Systems 7, 29. https:// doi.org/10.1186/1752-4458-7-29.

Shields LS, Pathare S, van der Ham AJ, Bunders J (2014). A review of barriers to using psychiatric advance directives in clinical practice. Administration and Policy in Mental Health 41, 753-766. https://doi.org/10.1007/s10488-013-0523-3.

Shields-Zeeman L, Pathare S, Walters BH, Kapadia-Kundu N, Joag K (2017). Promoting wellbeing and improving access to mental health care through community champions in rural India: the Atmiyata intervention approach. International Journal of Mental Health Systems 11, 6. https://doi.org/10.1186/ s13033-016-0113-3.

Slade M (2013). 100 ways to support recovery, 2nd Edition. Rethink Mental Illness. https://www.rethink.org/media/ 704895/100_ways_to_support_recovery_2nd_edition.pdf.

Slade M, Amering M, Oades L (2008). Recovery: an international perspective. Epidemiology and Psychiatric Sciences 17, 128-137.

Thara R, Rameshkumar TC (2012). Psychiatric advance directives: challenges of implementation. Indian Journal of Medical Ethics 9, 108. https://doi.org/10.20529/IJME.2012.032. 